\title{
Effects of nitrogen seeding on core ion thermal transport in JET ILW L-mode plasmas
}

\author{
N. Bonanomi ${ }^{1,2}$, P. Mantica ${ }^{2}$, J. Citrin ${ }^{3}$, C. Giroud ${ }^{4}$, E. Lerche ${ }^{5}$, C. Sozzi², \\ D. Taylor ${ }^{4}$, M. Tsalas ${ }^{3,4}$, D. Van Eester ${ }^{5}$ and JET contributors* \\ EUROfusion Consortium, JET, Culham Science Centre, Abingdon, OX14 3DB, UK \\ 1) University of Milano-Bicocca, Milano, Italy \\ 2) CNR- Institute of Plasma Physics "P. Caldirola", Milano, Italy \\ 3)DIFFER - Dutch Institute for Fundamental Energy Research, Eindhoven, The Netherlands \\ 4) Culham Centre for Fusion Energy, Abingdon, OX14 3DB, UK \\ 5)LPP-ERM/KMS, TEC partner, Brussels, Belgium
}

* See the author list of See the author list of "X. Litaudon et al., 2017 Nucl. Fusion 57, 102001"

\begin{abstract}
A set of experiments was carried out in JET ILW (Joint European Torus with ITER-Like Wall) L-mode plasmas in order to study the effects of light impurities on core ion thermal transport. $N$ was puffed into some discharges and its profile was measured by active Charge Exchange diagnostics, while ICRH power was deposited on- and off-axis in $\left({ }^{3} \mathrm{He}\right)-D$ minority scheme in order to have a scan of local heat flux at constant total power with and without $N$ injection. Experimentally, the ion temperature profiles are more peaked for similar heat fluxes when $N$ is injected in the plasma. Gyro-kinetic simulations using the GENE code indicate that a stabilization of Ion Temperature Gradient driven turbulent transport due to main ion dilution and to changes in $T_{e} / T_{i}$ and $s / q$ is responsible of the enhanced peaking. The quasi-linear models TGLF and QuaLiKiz are tested against the experimental and the gyro-kinetic results.
\end{abstract}

\section{Introduction}

One of the ways to reduce the heat loss deposition on the ITER (International Thermonuclear Experimental Reactor) divertor $[1,2]$ is to puff light impurities at the plasma edge. Furthermore, intrinsic light impurities, like $B e$ from the first wall, can enter in ITER plasmas. It is generally found that these impurities have peaked profiles [3, 4], so their impact on the core performances may not be negligible. On one hand, a stabilizing effect on turbulence is predicted by theory [5] and observed experimentally, as discussed below. On the other hand, impurities dilute the main ions thus reducing fusion power. Therefore, it is important to understand what their impact is on the main ion heat transport in the core, and validate existing models, in order to evaluate the trade-off between positive and negative effects and achieve optimal conditions for fusion.

In the last years, the effects of light impurity seeding on the plasma heat transport and energy confinement have been studied on different machines such as FTU [6, 7], TEXTOR-94 [8], ASDEX Upgrade [9], JET [8, 10], DIII-D [8], JT-60U [8] and C-mod [11, 12, 13]. In all these studies, that cover plasmas in Ohmic confinement mode, L-mode and H-mode, an increase of the plasma confinement in presence of light impurities has been observed. In most cases, a stabilization of the Ion Temperature Gradient (ITG) modes has been suggested to explain the improvement in the confinement. In some cases, especially in $\mathrm{H}$ mode, the stabilizing effect has been 
suggested to be more important at the plasma edge and on the plasma pedestal, with negligible effect of light impurities on the stabilization of the plasma core micro-instabilities [9]. In other studies, a direct role of light impurity seeding in the stabilization of micro-instabilities in the plasma core has been observed and studied $[6,7,8,9,11,12,13]$. At JET, a number of studies in H-modes indicate that $N$ seeding increases the plasma energy confinement time and helps to increase the performances with the ITER-Like wall (ILW) at the levels reached with the old Carbon wall $[10,14,15]$. These observations are linked with the stabilization effects of light impurities in the pedestal region, while no detailed studies of the effects of the light impurity on the core plasma region have been done yet in JET. This paper reports on a specific study of the effects of $N$ injection in the core of JET ILW L-mode plasmas, in which for the first time we determine separately the changes induced by the $N$ injection in the ITG threshold (i.e. the critical ion temperature $T_{i}$ inverse gradient length for ITG on-set) and in the ITG "stiffness" (i.e. the rate of increase of the ion heat flux with the $T_{i}$ inverse gradient length). We then compare such results with gyro-kinetic (GK) and quasi-linear (QL) models, thus providing a much more stringent validation test.

The paper is organized as follows. In section 2 the experimental set-up is described while in section 3 the numerical simulation set-up is presented. In section 4 the impact of $N$ on the plasma core heat transport is studied. A discussion on the results and the conclusions are presented in section 5.

\section{Experimental set-up}

The discharges studied in this paper were L-mode plasmas made in the JET tokamak (major radius $R_{0}=2.96 \mathrm{~m}$, minor radius $a=1 \mathrm{~m}$ ) with ILW. All plasmas are D plasmas with vacuum toroidal magnetic field $B_{T} \approx 3.3 T$, plasma current $I_{p} \approx 2 M A$, electron density $n_{2} \approx 3.5 \cdot 10^{19} \mathrm{~m}^{-3}$ and safety factor at the flux surface that encloses the $95 \%$ of the poloidal flux $q_{95} \approx 5$. The heating power consists of $3-4 \mathrm{MW}$ of ICRH (Ion Cyclotron Resonance Heating) using a ${ }^{3} \mathrm{He}$ minority concentration $n_{3}{ }_{\mathrm{He}} / n_{e} \approx 6 \%$, which ensures a dominant ion heating [16], and of 1.7-3 MW of NBI (Neutral Beam Injection), mainly to provide charge exchange measurements of $T_{i}$, rotation $\omega_{T}$ and impurity density $n_{Z}$ profiles. The ICRH power was deposited both on-axis $(R \approx 3.0 \mathrm{~m})$ and off-axis $(R \approx 3.45 \mathrm{~m})$ in order to obtain low and high values of ion heat flux $q_{i}$ for the study of $q_{i}$ versus $R / L_{T i}=-R_{0}\left|\nabla T_{i}\right| / T_{i}$ and its power deposition was evaluated with the PION code [17]. The ICRH power deposition to ions and the $T_{i}$ profiles for discharges n. 86749 (on-axis) and n. 86756 (off-axis) are shown in figure 1. The NBI power and its power deposition radial profile are similar in all the discharges. The NBI heating power on electrons and ions is calculated with the PENCIL code [18].

The measurement of the electron temperature $T_{e}$ is provided by the ECE (Electron Cyclotron Emission) diagnostic with an error of about $5 \%$, while $T_{i}$ and $\omega_{T}$ are measured by the active Charge-Exchange (CX) diagnostic with an error for $T_{i}$ of about $5-10 \%$, depending on the radial position, and for $\omega_{T}$ of about $10 \%$. The error on $T_{e} / T_{i}$ is about $9-12 \%$. The electron density $n_{e}$ is measured by high-resolution Thomson scattering (HRTS) with an uncertainty of about $15 \%$. Local values of $R / L_{T i}, R / L_{T e}$ and $R / L_{n}$ were obtained by local linear fits of $\ln \left(T_{i}\right), \ln \left(T_{e}\right)$ and $\ln \left(n_{e}\right)$ radial profiles averaged over a time interval $\Delta t \approx 1 s$. The fits are done using $r=\left(R-R_{i n}\right) / 2, R$ and $R_{i n}$ being the outer and inner radii of the flux surface on the magnetic axis plane, and averaging other multiple fits using a variable number of data points around the chosen radius (3-9 points). We drop the suffix 0 when indicating these quantities for convenience. The uncertainties on these parameters are then estimated by repeating the same procedure with different space intervals and evaluating the deviation in the set of values so obtained. Errors are typically $10-15 \%$ for $R / L_{T e / i}$ and $15-20 \%$ for $R / L_{n}$. The radial profile of the safety factor $q$ as well as the equilibrium plasma geometry are reconstructed by the EFIT equilibrium code with the MSE (Motional Stark Effect) or the Faraday rotation constraints. Typical errors on the safety factor are about $20 \%$.

The collisional exchange power density between ions and electron is calculated analytically as $p_{e i}=3 n_{e} m_{e} / M_{i}$. $\left(T_{e}-T_{i}\right) / \tau_{e}$, where $\tau_{e}$ is the electron collision time characterizing electron collisions with ions [19]. The radiated power density $p_{\text {rad }}$ has been taken into account but is negligible inside $r / a \sim 0.8$. The powers 
$P_{I C R H}, P_{N B I}, P_{e i}, P_{R A D}$ deposited within a flux surface are obtained integrating the power densities on the plasma volume and the ion heat fluxes are calculated in gyro-Bohm (GB) units as

$$
q_{i, g B}=\left[\left(P_{I C R H, i}+P_{N B I, i}+P_{e i}\right) / \Sigma\right] \cdot R^{2} /\left(n_{e} T_{e} \rho_{s}^{2} c_{s}\right)
$$

where $\Sigma$ is the flux surface, $c_{s}=\sqrt{T_{e} / M_{i}}, \rho_{s}=c_{s} M_{i} / e B_{T}$ and $M_{i}$ the main ion mass. Typical error on the heat fluxes is about $20 \%$ of the total flux. Turbulent flux threshold and stiffness at a chosen radial location are determined experimentally by quadratic fits on the diagrams of the GB normalized heat flux as a function of $R / L_{T}$ according to the heat flux parametrization proposed in $[20,21]$

$$
q_{i}=q_{i}^{r e s}+\chi_{s} \frac{n_{e} T_{e} c_{s} \rho_{s}^{2}}{R^{2}} \frac{R}{L_{T i}}\left(\frac{R}{L_{T i}}-{\frac{R}{L_{T i}}}^{\text {crit }}\right) \cdot \theta\left(\frac{R}{L_{T i}}-{\frac{R}{L_{T i}}}^{c r i t}\right)
$$

where $q_{i}^{\text {res }}$ is the residual flux not carried by the $R / L_{T i}$ driven instability, $\left(R / L_{T i}\right)^{\text {crit }}$ is the critical value of $R / L_{T i}$ above which the turbulent flux is driven by $R / L_{T i}, \chi_{s}$ is the stiffness coefficient and $\theta(\bullet)$ is the Heaviside function. From the curve of $q_{i, g B}$ versus $R / L_{T i}$, we identify $\left(R / L_{T i}\right)^{\text {crit }}$ as the intercept to zero flux (considering the residual flux to be negligible), whilst $\chi_{s}$ can be inferred from the slope of the curve. Equation 2.2 is a semi-empirical model called critical gradient model (CGM) originally proposed for the study of the electron heat transport. It assumes the existence of a critical temperature gradient length $L_{T}^{-1}=|\nabla T| / T$ for the turbulent transport and that the main drive of the transport over this critical value is $L_{T}^{-1}$. In this work we use the same formula for the ion heat flux, considering ITG modes, driven by $L_{T i}^{-1}$ over a critical threshold, the main source for the ion turbulent transport. This assumption is confirmed by the linear and non-linear simulations presented in section 4.4 of this work.

In order to study the effect of the presence of light impurities on the core heat transport, $N$ was puffed in discharges n. 86749-86756. The comparison between $\left(R / L_{T i}\right)^{\text {crit }}$ and $\chi_{s}$ with and without nitrogen is used to observe what kind of impact light impurities have on the core micro-instabilities. The density profiles of ${ }^{3} \mathrm{He}, \mathrm{Be}, \mathrm{N}$ have been measured by $\mathrm{CX}$, in order to obtain the concentrations and the peaking of the density of the light impurities in the plasma. The data analysis and the simulations are carried out at $\rho_{\text {tor }}=\sqrt{\left(\Phi / \pi B_{T}\right) /\left(\Phi / \pi B_{T}\right)_{\text {max }}}=0.33,0.5$, where $\Phi$ is the toroidal magnetic flux. The choice of these two radial positions is due to the changes in the ion temperature peaking observed experimentally and shown in section 4.3 and due to the different peaking of the $N$ density at these radii.

\section{$3 \quad$ Numerical simulation set-up}

The experimental ion heat fluxes are compared to gyro-kinetic simulations that calculate the turbulent part of the fluxes. Both linear and non-linear gyro-kinetic simulations have been carried out using the GENE (Gyrokinetic Electromagnetic Numerical Experiment) code [22, 23]. GENE solves the gyro-kinetic Vlasov equations coupled with the Maxwell equations within a $\delta f$ approximation [24] and using the field aligned coordinates $\{x, y, z\}$, where $z$ is the coordinate along the background magnetic field line, $x$ is the radial coordinate and $y$ is the binormal coordinate.

In all the simulations the flux-tube approximation and Miller geometry [25] were used, and collisions, external flow shear and finite- $\beta$ effects were included. Main ions, electrons and, when indicated, light impurity ions were retained as kinetic species. Typical grid parameters in the non-linear simulations were as follows: perpendicular box sizes $\left[L_{x}, L_{y}\right] \approx[190,125] \rho_{s}$, phase-space grid discretization $\left[n_{x}, n_{y}, n_{z}, n_{v \|}, n_{\mu}\right]=[128-256,24-64,32,32-$ $64,12]$, with $0.25-0.5 \leq k_{y} \rho_{s} \leq 1.6$, depending on the plasma parameters and on the number of kinetic species considered. The input parameters for the simulations are taken from discharge n. 86740 (without $N$ ) averaging over $9.5<t<10.5 s$ and from discharge n. 86749 (with $N$ ) averaging over $8<t<10 \mathrm{~s}$. The choice of these time intervals ensured convergence of the $q$ and $s$ profiles to a stable situation and the best CX measurements, as NBI notches (used for background signal subtraction in the CX analysis) are present in these intervals. The main plasma parameters for these discharges are reported in Table 1. In the gyro-kinetic nonlinear simulations 


\begin{tabular}{|c|c||c|c|c|c|c|c|c|c|c|c|}
\hline$\#$ & $\rho_{\text {tor }}$ & $s$ & $q$ & $R / L_{\text {ne }}$ & $R / L_{T e}$ & $R / L_{\text {Ti }}$ & $Z_{\text {eff }}$ & $T_{e} / T_{i}$ & $\nu_{\text {eff }}$ & $n_{N}$ & $R / L_{N}$ \\
\hline \hline \multirow{2}{*}{86740} & 0.33 & 0.4 & 1.5 & 2.8 & 7.9 & 4.2 & 1.4 & 0.9 & 0.08 & $0 \%$ & - \\
\cline { 2 - 13 } & 0.5 & 0.8 & 1.9 & 2.8 & 7.8 & 5 & 1.4 & 0.86 & 0.17 & $0 \%$ & - \\
\hline \hline \multirow{2}{*}{86749} & 0.33 & 0.35 & 1.35 & 2.8 & 8 & 4.5 & 1.9 & 0.9 & 0.1 & $1.2 \%$ & 2.0 \\
\cline { 2 - 13 } & 0.5 & 1.1 & 1.6 & 2.9 & 8.0 & 6 & 1.9 & 0.74 & 0.2 & $1.2 \%$ & 0.5 \\
\hline
\end{tabular}

Table 1: Main plasma parameters of the studied discharges at $\rho_{\text {tor }}=0.33,0.5$. Here $\nu_{e f f}=0.1 \cdot Z_{e f f} n_{e} / T_{e}^{2}$.

just one kinetic light impurity species $(N)$ has been used and $Z_{\text {eff }}=1.0$ has been assumed in simulations of discharge n. 86740 without $N$, while $Z_{\text {eff }}=1.5$ has been used in the simulations of shot n. 86749 with $N$. This choice has been made to save computational time and considering that the changes in the plasma are mainly due to $N$ puffing, the other light impurities being present in all the discharges at the same concentrations $\left(\mathrm{Be} \sim 1 \%,{ }^{3} \mathrm{He} \sim 6 \%\right)$.

The standalone versions of the quasi-linear models TGLF [26, 27] and QuaLiKiz [54, 28, 29] have also been tested against the experimental results and the non-linear gyro-kinetic simulations. The TGLF simulations have been carried out in Miller geometry and the well established 'sat0' saturation rule has been adopted. QuaLiKiz instead features only the $s-\alpha$ geometry. While Miller geometry takes into account the real shape of the flux surfaces though parameters such as triangularity and elongation, the $s-\alpha$ geometry considers simple circular flux surfaces and the Shafranov shift. This can have an impact on the predicted turbulent state, as parameters such as elongation have a stabilizing effect on the micro-instabilities. The same input parameters and settings (finite- $\beta$, external flow shear, $k_{y} \rho_{s}$ range and $n_{y}$, number of kinetic species) used in the GENE gyro-kinetic simulations have been used in the quasi-linear simulations.

\section{Light impurity effects on thermal transport}

\subsection{Predict-first simulations}

Before showing the experimental results and the related numerical simulations, we present a series of linear and non-linear gyro-kinetic simulations made before the experiment, in a predict-first approach, in order to evaluate the magnitude of the effects to be expected in experimental conditions similar to ours. These simulations have been carried out with the GENE code in flux-tube approximation and using input parameters from the JET discharge n. 73221 at $\rho_{\text {tor }}=0.33$. This discharge had similar settings and plasma parameters as the discharges studied in this work (L-mode, same $B_{t}, I_{p}, n_{e}, q_{95}, 1.6 \mathrm{MW}$ of NBI and $3 \mathrm{MW}$ of ICRH on ions using $6 \%$ of ${ }^{3} \mathrm{He}$ ), but was done with the old Carbon-wall. In these simulations, kinetic electrons, kinetic deuterium and a third kinetic ion species $(N, N e$ or $A r)$ have been used. The geometry parameters have been taken from a CRONOS [30] simulation of the discharge as described in [31]. The results from the simulations are shown in figures 2 and 3. As can be seen, linear simulations predict a stabilization of ITG modes in presence of light impurities. The level of the stabilization depends, for the same value of $Z_{e f f}$, on the main ion dilution (in this case there is more dilution using $N$ ) and on the value of $R / L_{n, Z}$ of the light impurity. An increase in the main ion dilution (and of $Z_{\text {eff }}$ ) results in an increase of the stabilization effects. An increase in the value of the normalized impurity density gradient results in a stronger stabilizing effect: with $R / L_{n, Z} \approx 0$ there is a reduction of the ion temperature stiffness but no substantial increase of the ITG threshold, while for higher values of $R / L_{n, Z}$ an important increase of the ITG critical threshold is visible. Non-linear simulations confirm what found in the linear simulations. Depending on the level of the main ion dilution and on the peaking of the light impurity species, a stabilization of the ITG turbulent ion heat fluxes is observed.

The simulations predict that, in order to observe a strong effect of the light impurity on the ion thermal transport, $Z_{\text {eff }}$ and the main ion dilution are key factors. At the same level of $Z_{\text {eff }}$, the bigger effect between $N, N e$ and $A r$ is due to $N$ due to higher main ion dilution. For these reasons, in the experiment carried out at JET and studied in this work, $N$ puffing has been chosen. Furthermore, from the non-linear simulations, a 
value or $R / L_{n, N} \approx 1.4$ is expected, with the considered plasma parameters, in stationary conditions (zero $N$ flux). With this value of $R / L_{n, N}$, a change in $Z_{\text {eff }}$ of at least 1 is expected to be necessary in order to observe a visible effect on the ion thermal transport outside error bars.

\subsection{Comparison between C-Wall and ITER-Like Wall L-mode plasmas}

The validity of the predict-first study of sect. 4.1, based on the parameters of a reference C-wall discharge without $N$, for planning ILW discharges to study the $N$ effect, relies on the expectation that core plasma transport and local plasma parameters do not depend on the first wall material for L-mode plasmas. This has been indeed verified when L-mode plasmas in ILW with similar experimental settings as in C-wall have become available. When the same settings for $B, I_{p}, q_{95}, n_{e 0}$, shape, and heating schemes (low NBI power and ICRH in $D-{ }^{3} \mathrm{He}$ minority scheme) are used, no substantial differences are observed between C-Wall and ILW in the core ion heat turbulent transport. In figure $4 \mathrm{a}$, the $T_{i}$ profiles from discharges $\mathrm{n}$. 78829 (C-Wall with 3.7 MW of NBI and 2.5 MW of on-axis ICRH heating) and n. 86740 (ILW with $3 \mathrm{MW}$ of NBI and $3 \mathrm{MW}$ of on-axis ICRH heating) are shown. In figure $4 \mathrm{~b}$ also a comparison between $q_{i, g B_{s}}\left(R / L_{T i}\right)$ in C-Wall and ILW for discharges studied in the past [see for example [32,33]], the discharges with no $N$ studied in this paper and other two ILW discharges (n. 90668, 90671) is shown. No substantial differences in the experimental profiles, in the experimental threshold of the ion heat turbulent transport and in the ion stiffness have been observed. In fact, the significant degradation of ILW confinement with respect to C-wall reported e.g. in [34] was found only in H-modes and mainly due to a pedestal reduction, with the core transport substantially unchanged [35].

\subsection{Experimental observations}

In this section, the main differences between discharges with and without $N$ in JET ILW L-modes are presented. The comparison has been made between shot n. 86740 , with no $N$, and shot n. 86749 , with $n_{N} / n_{e} \approx 1.2 \%$, averaging over the same time intervals used for the gyro-kinetic simulations. The measured concentration of $N$ was constant over the chosen time period. For the heat flux scans, also discharges n. 86746, with off-axis ICRH heating and no $N$, and n. 86756, with off-axis ICRH heating and $n_{N} / n_{e} \approx 1.2 \%$, have been used. This was close to the maximum level of $N$ that could be puffed into the plasma without causing a disruption. The radial profiles of the temperatures and of the electron density of the two discharges as well as the measured density profile of $N$ (magnified by a factor of 75 to be more visible in the plot) in discharge 86749 are shown in figure 5. The level of $N$ reached in the plasma is not predicted by gyro-kinetic simulations to be high enough to have the desired change of at least 1 in $Z_{\text {eff }}$ and the density peaking of $N$ changes along the radius, the profile being peaked inside $\rho_{\text {tor }} \sim 0.4$ and outside $\rho_{\text {tor }} \sim 0.7$ and flat for $0.4 \lesssim \rho_{\text {tor }} \lesssim 0.7$ (see also [4]). Considering the gyrokinetic simulations, no substantial differences outside error bars are then expected. Nevertheless the profiles of $T_{i}$ and $T_{e}$ are more peaked in the discharge with $N$ puff and a stabilization of the ion heat turbulent transport can be observed in discharges with $N$ puff with respect to discharges with no $N$, especially at $\rho_{\text {tor }}=0.5$. This can be seen from the shift of the experimental ion temperature gradient, well outside error bars at $\rho_{\text {tor }}=0.5$, in the ion heat flux scans obtained using on and off-axis ICRH heating at $\rho_{\text {tor }}=0.33,0.5$ and shown in figure 6. No visible effect on the ion stiffness is observed outside error bars.

A change in $Z_{\text {eff }}$, from $Z_{\text {eff }} \approx 1.4$ in the discharges without nitrogen to $Z_{\text {eff }} \approx 1.9$ in the discharges with nitrogen puff, has been observed. Also, as the electron density has been kept the same and the other light impurities have the same measured concentrations ( $\sim 1 \%$ of $B e$ and $\sim 6 \%$ of ${ }^{3} \mathrm{He}$, they have also the same density profiles between the discharges), there is a major dilution of the main ion $(D)$ species in the discharges with nitrogen (from $n_{D} / n_{e} \sim 84 \%$ to $n_{D} / n_{e} \sim 75 \%$ ). As a consequence of the change of $Z_{\text {eff }}$, also the radial profile of the plasma resistivity $\eta_{p}$ changes. This leads to a change in the radial profile of the plasma current density and, consequently, to a change in the safety factor $q$ and in the magnetic shear $s$. The comparisons of the radial profiles of the plasma resistivity and of $s / q$ between shots with and without nitrogen are shown in figure 7. Another difference between discharges with and without $N$ puff is the ratio $T_{e} / T_{i}$, as can be seen in 
figure 5. The electron density, the electron density peaking and the edge temperatures do not show substantial changes that could explain the change in the temperature profiles.

\subsection{Numerical study}

In order to investigate the causes of the described changes in the plasma due to the presence of nitrogen, linear and non-linear gyro-kinetic simulations have been carried out using the code GENE as described in section 3 . At $\rho_{\text {tor }}=0.33$ the radial density profile of $N$ is peaked $\left(R / L_{n_{N}}=2\right.$ has been used in the simulations) and the differences in $T_{e} / T_{i}$ and $s / q$ between cases without and with $N$ are small. At $\rho_{t o r}=0.5$ the $N$ radial density profile is flat $\left(R / L_{n_{N}}=0.5\right.$ in the simulations) and the differences in $T_{e} / T_{i}$ and $s / q$ are more substantial. The observed changes in $Z_{e f f}, n_{D}, s / q$ and $T_{e} / T_{i}$ are all predicted to play a role in the stabilization of the ion heat flux $[5,36,37]$.

In figure 8 , a study on the effects of the various impurities in the plasma using linear gyro-kinetic simulations and data from discharge n. 86749 at $\rho_{\text {tor }}=0.5$ as input, is shown. The effects of the different impurities in the plasma, using the experimental concentrations and density peaking, on the linear growth rate of the dominant instability is shown on both ion and electron scale modes. From the simulations it is clear that the strongest effect is due to nitrogen and that its effect is stronger with higher impurity density gradients, as expected. Furthermore, a linear scan in $R / L_{T i}$, fixing $k_{y} \rho_{s}=0.33$ in the simulation, is shown. These simulations confirm what found in the predict-first study made using the C-Wall discharge.

A study with linear gyro-kinetic simulations on the effect of the changes in the plasma parameters described in section 4.3 has been carried out at $\rho_{\text {tor }}=0.5$ and the results are shown in figure 9 . In these simulations the parameters from discharge n. 86740 have been used first (black lines and circles in figure 9). In all the simulations, the main instabilities up to $k_{y} \rho_{s} \approx 0.7$ are the ITG modes (the real part of the frequency has the same sign of the ion diamagnetic drift), while, at higher values of $k_{y} \rho_{s}$, TEM/ETG modes are the dominant ones. Changing the values of $s / q$ and $T_{e} / T_{i}$ to the values of discharge $n .86749$ leads to a reduction of the ITG growth rate of $\sim 25 \%$ at $k_{y} \rho_{s}=0.4$. Adding also $1.2 \%$ of $N$, using $R / L_{N}=0.5$, leads to a further reduction of $\sim 12 \%$. So, linearly, the stronger stabilizing effect on ITGs are predicted to be related to the changes in $T_{e} / T_{i}$ and in $s / q$ in these discharges (figure $9 \mathrm{a}$ ). This stabilization leads to a change in the ITG $R / L_{T i}$ threshold and is expected to be the main stabilizing mechanism of the ion heat flux. This can also be seen from the nonlinear ion heat flux spectra in $k_{y} \rho_{s}$ shown in figure 9c. The main contribution to the ion heat flux is coming from $0.2 \leq k_{y} \rho_{s} \leq 0.4$, where the ITG modes are dominating. Furthermore, stabilizing effects due to higher $Z_{\text {eff }}$ and $s$ on TEM and due to higher $\tau=Z_{e f f} \cdot T_{e} / T_{i}$ and $s / q$ on ETG modes have been found and can contribute to the reduction of the ion heat flux, but their effect is less important with respect to the stabilization of ITG modes in our case.

The ion heat fluxes from the nonlinear simulations are shown in figure 6 and compared with the experimental fluxes. The non-linear simulations were able to reproduce quite well both the electron and the ion heat flux experimental levels, confirming that the changes in the plasma parameters, obtained when $N$ has been puffed into the plasma, lead to an overall stabilizing effect of the turbulent heat transport. At $\rho_{\text {tor }}=0.33$, the changes in $s / q$ and $T_{e} / T_{i}$ being small, only the effect of the main ion dilution has a significant role but, as the amount of nitrogen injected in the plasma and the changes induced in $Z_{\text {eff }}$ were rather low (higher values causing disruptions), the predicted stabilization is not enough to be visible outside the experimental error bars. At $\rho_{\text {tor }}=0.5$, an increase of the threshold of the turbulent transport as well as a little reduction of the ion stiffness are predicted by the simulations and follow quite well the experimental points. The increase of the threshold in this case is mainly due to the changes of $T_{e} / T_{i}$ and $s / q$, while the reduction of the stiffness level is due to the main ion dilution effect due to the presence of $N$ with rather flat local profile. This can be seen comparing the black full circles and the blue full triangles in figure $6 \mathrm{~b}$. The blue triangles indicate simulations of discharge $\mathrm{n}$. 86740 where $2 \%$ of $N$ with $R / L_{N}=0.5$ has been added artificially in order to study the pure effect of $N$. No big changes in the threshold have been observed in this case, but a clear reduction of the ion stiffness.

As explained in section 3, the stand-alone versions of the quasi-linear codes TGLF and QuaLiKiz have been 
tested against non-linear gyro-kinetic simulations and experiments. The results are shown in figure 10 and in figure 11. TGLF tends to underestimate the ion stiffness at both radii, while QuaLiKiz reproduces it quite well, although the predicted critical threshold is a little shifted between GENE and QuaLiKiz. Both codes show an overall effect of the changes in $Z_{e f f}, n_{D}, s / q$ and $T_{e} / T_{i}$ comparable in magnitude to the experimental observations and with the gyro-kinetic results. When the pure effect of $N$ is considered (using $N=2 \%$ and $R / L_{n, N}=0.5$ ), as can be seen in figure 10c while GENE and TGLF predict an effect primarily on the ion stiffness, QuaLiKiz predicts an upshift of the threshold but no effects on the stiffness. As QuaLiKiz has been implemented in $s-\alpha$ geometry, we made a comparison also between QuaLiKiz, TGLF and GENE using shifted circular geometry. This comparison is shown figure 11b. When circular geometry is used, an important increase in ion stiffness in observed in GENE and TGLF (TGLF still underestimating ion stiffness with respect to GENE as in Miller geometry). Also QuaLiKiz is now significantly below the GENE $s-\alpha$ results.

\section{Discussion and conclusions}

A detailed study of the effect of $N$ seeding on the core ion thermal transport of JET L-mode ILW plasmas has been carried out. A predict-first approach based on gyro-kinetic simulations with parameters from reference shots in C-wall was very useful to guide the experimental choice of the seeding gas and of the level of injection to ensure detectability of the effects. From these simulations a change in $Z_{\text {eff }}$ of at least 1 was predicted to impact the experimental ion heat flux enough to be able to observe this impact outside experimental uncertainties. High values of the light impurity density gradient are also expected to increase the stabilizing effects. When executing the experiment, a concentration lower than desired was reached due to high disruptivity. In addition, the $N$ profile at mid radius turned out to be flatter than theoretically predicted (as discussed in [4]). These two unforeseen circumstances led the experiment into a situation where the predicted impurity effect on the ion thermal transport would be inside the experimental uncertainties. Still, a remarkable increase of the ion temperature peaking was observed, well outside uncertainties. Gyro-kinetic simulations using the actual parameters of the discharges with and without $\mathrm{N}$ show a stabilization of the ion heat transport when $\mathrm{N}$ is puffed into the plasma. This is related to changes in the plasma parameters induced by the $\mathrm{N}$ puff, such as main ion dilution, $s / q$ and $T_{e} / T_{i}$, with the changes in $s / q$ and $T_{e} / T_{i}$ being the dominant mechanisms leading to the observed ITG critical threshold upshift. This explains why the data show mainly a threshold upshift, rather than a decrease in stiffness as would be expected for dilution in presence of a rather flat impurity profile. The effect of dilution itself, although alone not enough to give an effect on $T_{i}$ outside uncertainties, is anyway inducing a change of $T_{i} / T_{e}$ that produces further ITG stabilization, which together with the $q$ profile change eventually leads to a well observable $T_{i}$ peaking.

How much the effects that were observed in these experiments will extrapolate to high power scenarios in JET or ITER is difficult to assess. It is however clear that any prediction of a scenario with impurities will have to account in an integrated approach for both the direct effects of main ion dilution and of change in $Z_{\text {eff }}$ and the secondary effects due to changes in $T_{i} / T_{e}$ or $q$ profile, which can play an important role, as in the experiments described here. It is therefore important to test and improve models in order to have reliable tools for such integrated modelling. This was the main aim of the work presented in this paper. Gyro-kinetic simulations were found to reproduce the experimental observations pretty well, considering experimental uncertainties. We then tested TGLF (sat0) and QuaLiKiz against our experimental observations and also against nonlinear gyrokinetic simulations in a broader range of $N$ concentrations. TGLF tends to generally underestimate the ion stiffness, but concerning the impurity effects it is able to reproduce quantitatively the effects on both threshold and stiffness observed in gyro-kinetic runs. QuaLiKiz reproduces much better the ion stiffness, but shows critical thresholds slightly shifted respect to GENE. Also, the predicted effects of light impurities on ITGs from QuaLiKiz is mainly a shift of the critical $R / L_{T i}$ threshold, quantitatively of the right magnitude, but with no effect on stiffness at variance with GENE and TGLF. The effect of geometry has also been found important. When the same geometry $(s-\alpha)$ is used in GENE nonlinear simulations as in QuaLiKiz, the GENE heat flux 
increases significantly and the shift between their predicted thresholds in $R / L_{T i}$ is higher. These results call for an improvement of both QL models, particularly on the match of the ion stiffness and threshold already in the case with no $N$, whilst the effect of $N$ overall is adequately modelled.

Concerning the question if overall the $N$ seeding leads to poorer or better fusion performance, this depends on whether the enhancement of the ion temperature and so of the fusion reaction rate wins over the dilution of the fuel ion density. This depends strongly on the plasma conditions. A factor $\alpha<1$ that multiplies the D and $\mathrm{T}$ densities (supposing for simplicity that $n_{D}=n_{T}$ and that the dilution is constant on the plasma profile) can reduce the power from the reactions by $\left(1-\alpha^{2}\right) \%$ (in our case $\sim 16 \%$ ), but an increase in the ion temperature by $\sim 1 \mathrm{keV}$, as we observe in these experiments, can raise the power more strongly (in our case $\sim 300 \%$ ). The difference is that the loss due to the main ion dilution is always the same independently of the plasma density, while the gain due to an increase of the ion temperature strongly depends on the plasma temperature. Between 10 and $14 \mathrm{keV}$, the increase in the fusion reaction rate is still of $\sim 20 \% / \mathrm{keV}$, but for higher temperatures the increase drops quickly. Other considerations on the kind of impurity puffed into the plasma (most probably $A r$ for ITER) and on the peaking of the impurity density profile should be taken into account, since linear and non-linear gyro-kinetic simulations predict that the ITGs stabilization is stronger with increasing density peaking of the light impurity. In particular, when the impurity density gradient is close to, or lower than, zero, the predicted effect is mainly a reduction of the ion temperature stiffness, while, when the gradient of the light impurity density increases, the dominant effect is an increase of the ITGs critical threshold in $R / L_{T i}$.

\section{Acknowledgement}

The authors would like to thank Emiliano Fable and Gary Staebler for precious suggestions and discussions on the use of the TGLF model. The authors are grateful to D. R. Mikkelsen for assistance. This research used resources of the National Energy Research Scientific Computing Center, a DOE Office of Science User Facility supported by the Office of Science of the U.S. Department of Energy under Contract No.DE--AC02--05CH11231. A part of this work was carried out using the HELIOS supercomputer system at Computational Simulation Centre of International Fusion Energy Research Centre (IFERC--CSC), Aomori, Japan, under the Broader Approach collaboration between Euratom and Japan, implemented by Fusion for Energy and JAEA. We acknowledge the CINECA award under the ISCRA initiative, for the availability of high performance computing resources and support. This work has been carried out within the framework of the EUROfusion Consortium and has received funding from the Euratom research and training programme 2014-2018 under grant agreement No 633053. The views and opinions expressed herein do not necessarily reflect those of the European Commission.

\section{References}

[1] Mandrekas J., Stacey W. M., Nucl. Fusion 35, 843 (1995).

[2] Pacher G. W., et al., Nucl. Fusion 47, 469 (2007).

[3] Manas. P. et al, $42^{\text {nd }}$ EPS conference on plasma physics, Lisbon, Portugal (2015).

[4] N. Bonanomi et al., "Light impurity transport in JET plasmas" (2017), submitted to Nucl. Fusion.

[5] Dominguez R. R. and Rosenbluth M. N., Nucl. Fusion 29, 844 (1989).

[6] M. Romanelli et al., Nucl. Fusion 51, 103008 (2011).

[7] G. Szepesi et al., Nucl. Fusion 53, 033007 (2013). 
[8] Ongena J., et al., Phys. Plasmas 8, 2188 (2001).

[9] Tardini G. et al., Plasma Phys. Control. Fusion 55, 015010 (2013).

[10] Giroud C., et al, Plasma. Phys. Control Fusion 57, 035004 (2015).

[11] Porkolab M. et al., Plasma Phys. Control. Fusion 54, 124029 (2012).

[12] Ennever P., et al., Phys. Plasmas 22, 072507 (2015).

[13] Porkolab M., et al., 26 ${ }^{\text {th }}$ IAEA Fusion Energy Conference, Kyoto, Japan (2016).

[14] Maddison G. P., et al., Nucl. Fusion 54, 073016 (2014)

[15] Jaervin A. D., et al., Nucl. Fusion 56, 046012 (2016)

[16] Van Eester D., et al., Plasma Phys. Control. Fusion 51, 044007 (2009).

[17] Eriksson L. G., Hellsten T. and Willén U., Nucl. Fusion 33, 1037 (1993).

[18] Challis C., et al., Nucl. Fusion 29, 563 (1989).

[19] Wesson J. and Campbell D. J., 'Tokamaks', Oxford University Press, $4^{\text {th }}$ edition (2011).

[20] Cenacchi G. and Taroni A., Rapporto ENEA RT/TIB/88/5 (1998).

[21] F, Imbeaux, F. Ryter. and X. Garbet., Plasma Phys. Control. Fusion 43, 1503 (2001).

[22] Jenko F., et al., Phys. Plasmas 7, 1904 (2000).

[23] Görler T., et al., J. Comput. Phys. 230, 7053 (2011).

[24] Brizard A. J., Hahm T. S., Rev. Mod. Phys. 79, 421 (2007).

[25] Miller R. L., et al., Phys. Plasmas 5, 973 (1998). 
[26] Staebler G. M., Kinsey J. E. and Waltz R. E., Phys. Plasmas 12, 102508 (2005)

[27] Staebler G. M., Kinsey J. E. and Waltz R. E., Phys. Plasmas 14, 055909 (2007)

[54] Bourdelle C., et al, Phys. Plasmas 14, 112501 (2007)

[28] Bourdelle C., et al., Plasma Phys. Control. Fusion 58, 014036 (2016)

[29] J. Citrin et al., accepted to Plasma Phys. Control. Fusion (2017), https://doi.org/10.1088/1361$6587 /$ aa8aeb.

[30] Artaud J. F., et al., Nucl. Fusion 50, 043001 (2010).

[31] Citrin J., et al., Nucl. Fusion 54, 023008 (2014).

[32] P. Mantica et al., Phys. Rev. Lett. 107, 135004 (2011).

[33] P. Migliano et al., Plasma Phys. Control. Fusion 55, 055003 (2013).

[34] M. N. A. Beurskens et. al., Nucl. Fusion 54, 043001 (2014).

[35] Hyun-Tae Kim et. al., Plasma Phys. Control. Fusion 57, 065002 (2015).

[36] Guo S. C. and Romanelli F., Phys. Fluids B 5 (2), 520 (1993).

[37] Migliano P., et al., Plasma Phys. Control. Fusion 55, 055003 (2013).

[38] Bonanomi N., et al., Nucl. Fusion 55, (2015).

[39] Peeters A. G., et al., Phys. Plasmas 12, 022505 (2005).

[40] Ryter F., et al., Phys. Rev. Lett. 95, 085001 (2005) 


\section{FIGURES}

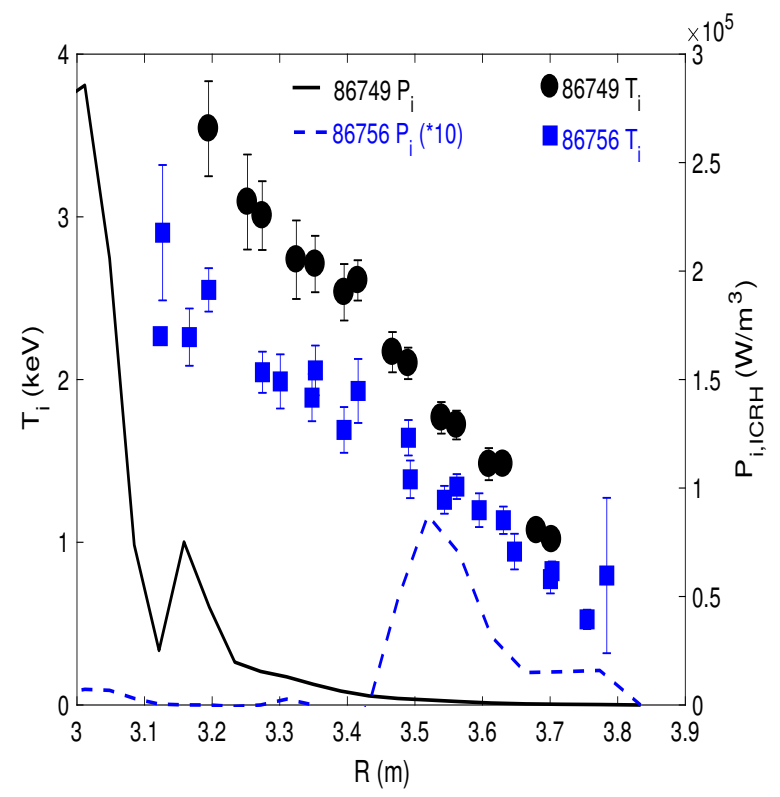

Figure 1: ICRH power deposition and ion temperature of discharge n. 86749 (on-axis ICRH) and n. 86756 (off-axis $\mathrm{ICRH}$ )

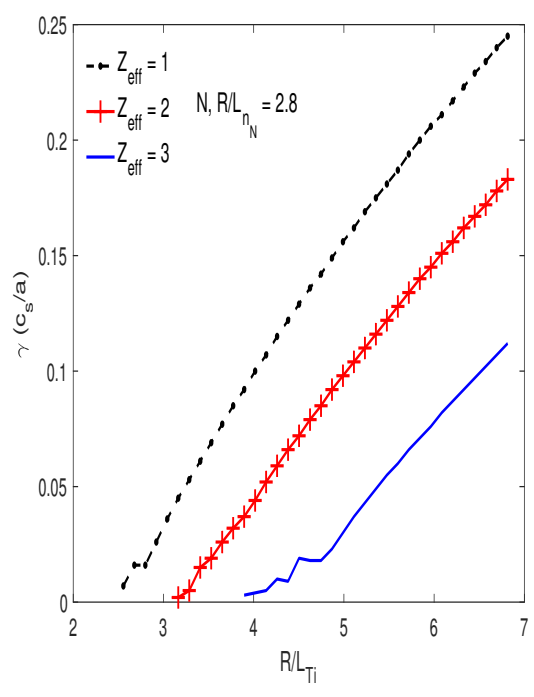

b)

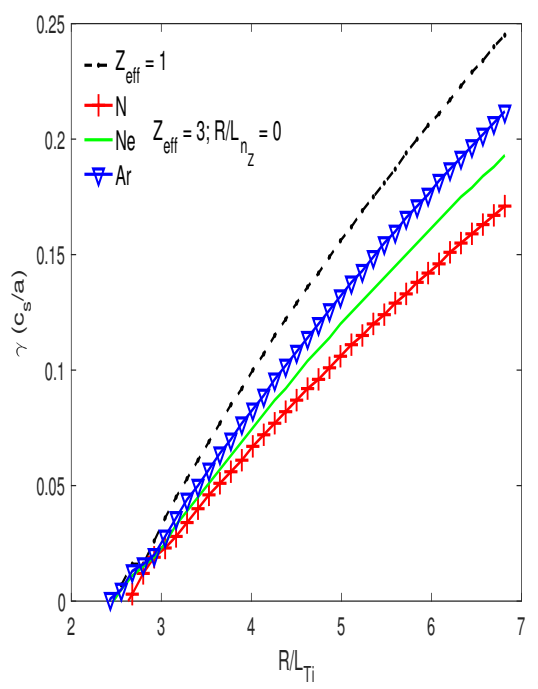

c)

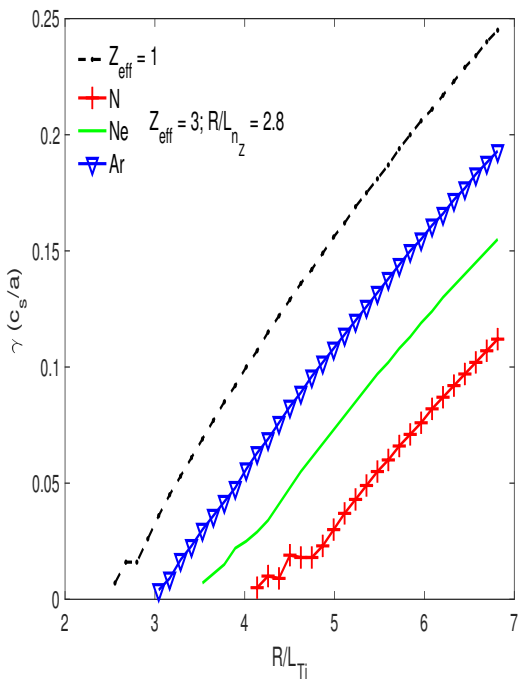

Figure 2: Normalized linear growth rate $\gamma$ of the main micro-instability in the simulation as a function of $R / L_{T i}$ from linear gyro-kinetic simulations at $\rho_{\text {tor }}=0.5$. Effect of $N$ at different concentrations using $R / L_{n, Z}=2.8$ (a), effects of $N, N e$ and $A r$ at the same level of $Z_{\text {eff }}=3$ and using $R / L_{n, Z}=0$ (b) and effects of $N, N e$ and Ar at the same level of $Z_{\text {eff }}=3$ and using $R / L_{n, Z}=3$ (c). Black dotted lines indicate the case with no impurities $\left(Z_{\text {eff }}=1\right)$. 

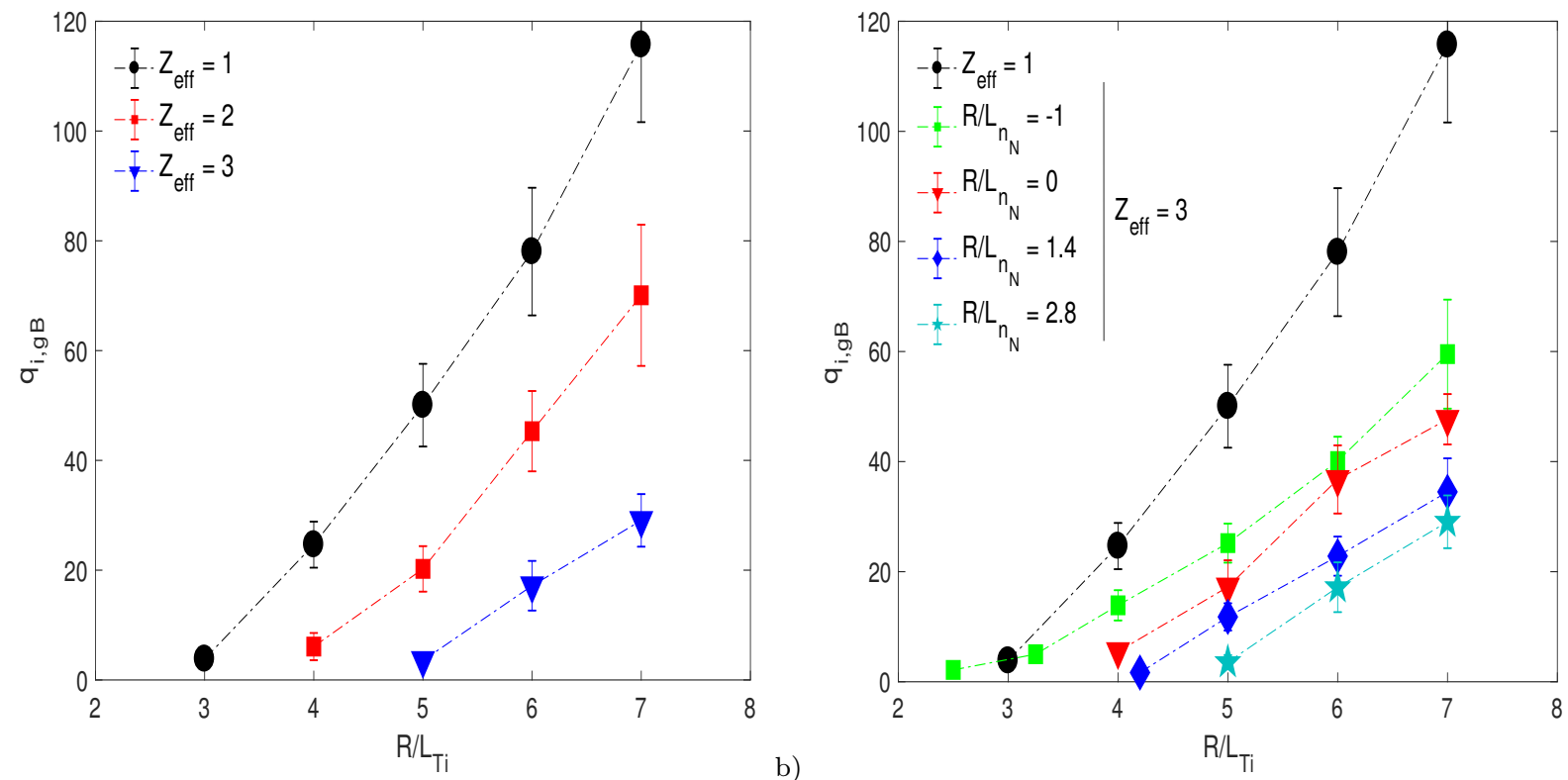

Figure 3: Normalized ion heat flux $q_{i, g B}$ from non-linear gyro-kinetic simulation as a function of $R / L_{T i}$. Effects of $N$ at different levels of $Z_{\text {eff }}$ and using $R / L_{n, N}=2.8$ (a) and effects of $N$ at the same level of $Z_{\text {eff }}=3$ but using different values of $R / L_{n, N}$ (b). Black circles indicate the case with no impurities $\left(Z_{\text {eff }}=1\right)$. 


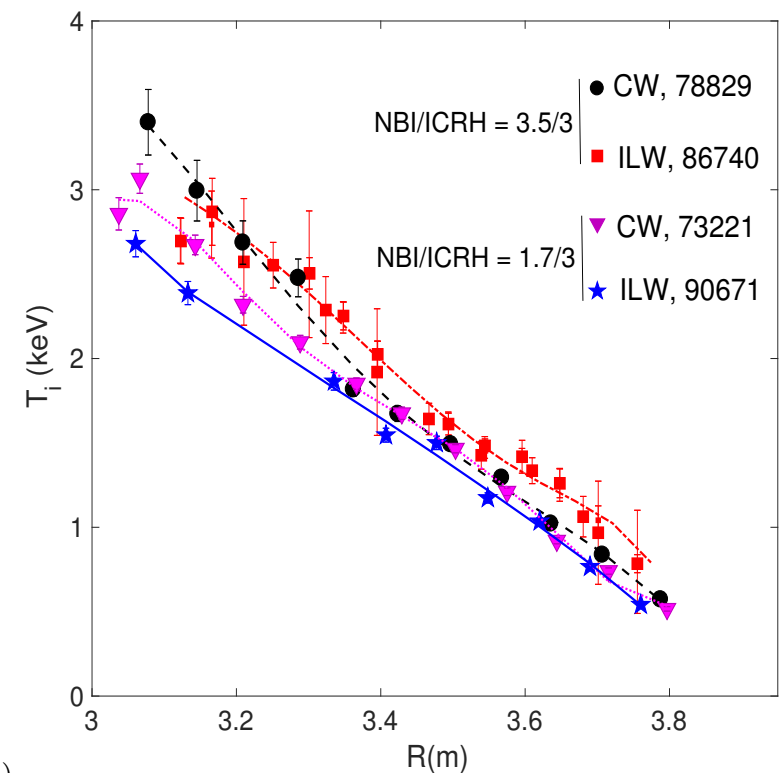

a)
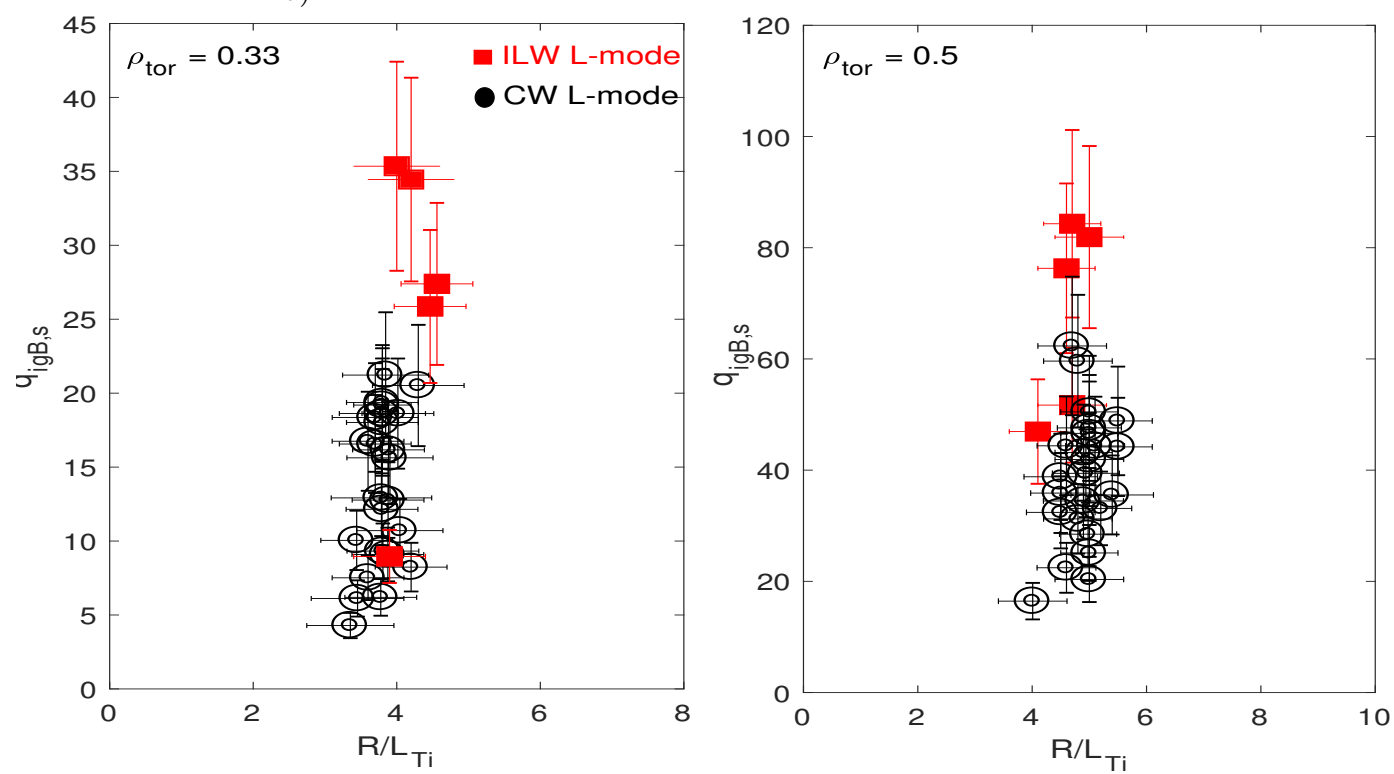

b)

Figure 4: a)Comparison of ion temperature profiles obtained in JET CW and ILW using similar experimental settings $\left(n_{e}, I_{p}, q, B_{t}\right)$ and heating scheme. b)Comparison of $q_{i, g B}\left(R / L_{T i}\right)$ in JET CW and ILW plasmas. 

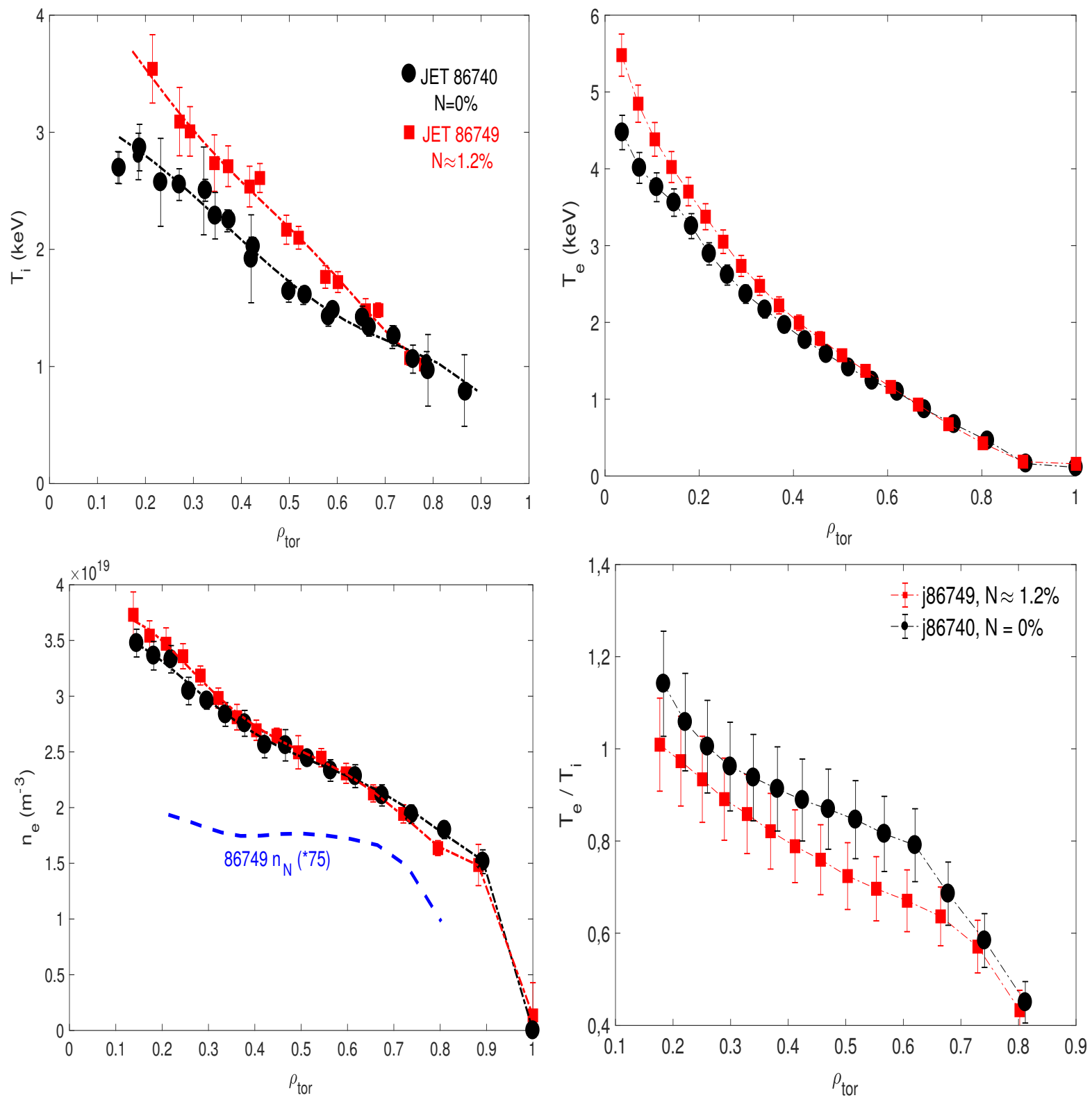

Figure 5: Radial profiles of the ion temperature $T_{i}$, electron temperature $T_{e}$, electron density $n_{e}$ and $T_{e} / T_{i}$ of $J E T$ discharges $n .86740$ at $t=10 \mathrm{~s}$ (black circles) and $n .86749$ at $t=9 \mathrm{~s}$ (red squares). Discharge $n .86740$ had no $N$, while discharge $n$. 86749 had a concentration of nitrogen $n_{N} / n_{N} \approx 1.2 \%$. 

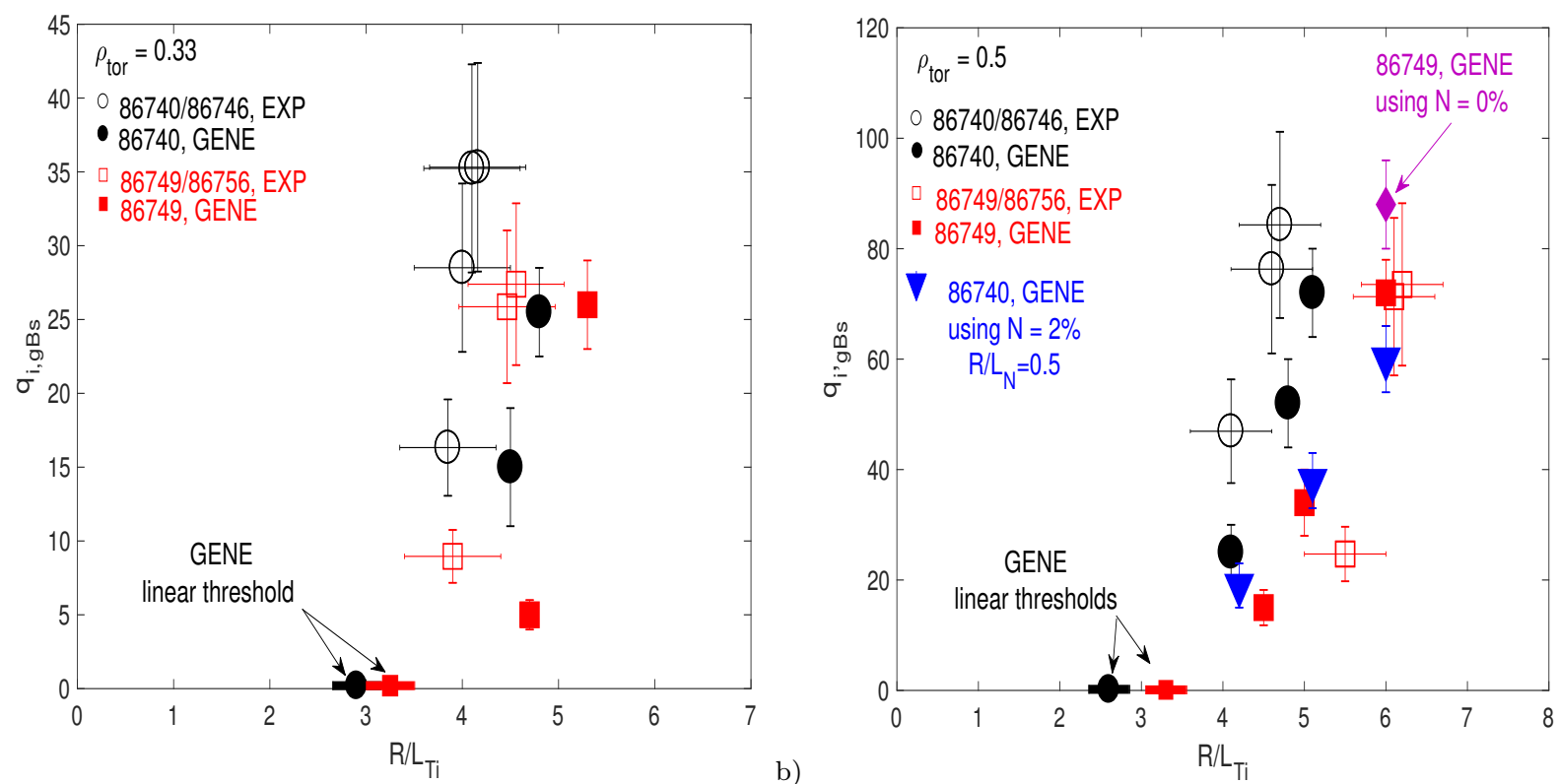

Figure 6: $q_{i, g B s}\left(R / L_{T i}\right)$ at $\rho_{\text {tor }}=0.33$ (a) and at $\rho_{\text {tor }}=0.5$ (b). The empty black circles represent the experimental results using $n_{N} / n_{e}=0 \%$ from discharges $n .86740$ (on-axis ICRH) and n. 86746 (off-axis ICRH), while the full black circles represent the results from nonlinear gyro-kinetic simulations. The empty red squares represent the experimental results using $n_{N} / n_{e} \approx 1.2 \%$ from discharges $n$. 86749 (on-axis ICRH) and n. 86756 (off-axis ICRH), while the full red squares represent the results from nonlinear gyro-kinetic simulations. In figure (b), the blue triangles in figure $8 \mathrm{~b}$ represent gyro-kinetic simulations of shot n. 86740 adding $N=2 \%$ and using $R / L_{N}=0.5$, while the purple diamond represent a gyro-kinetic simulation of shot $n .86749$ but using $N=0 \%$. The gyrokinetic simulations results are discussed in section 4.4.
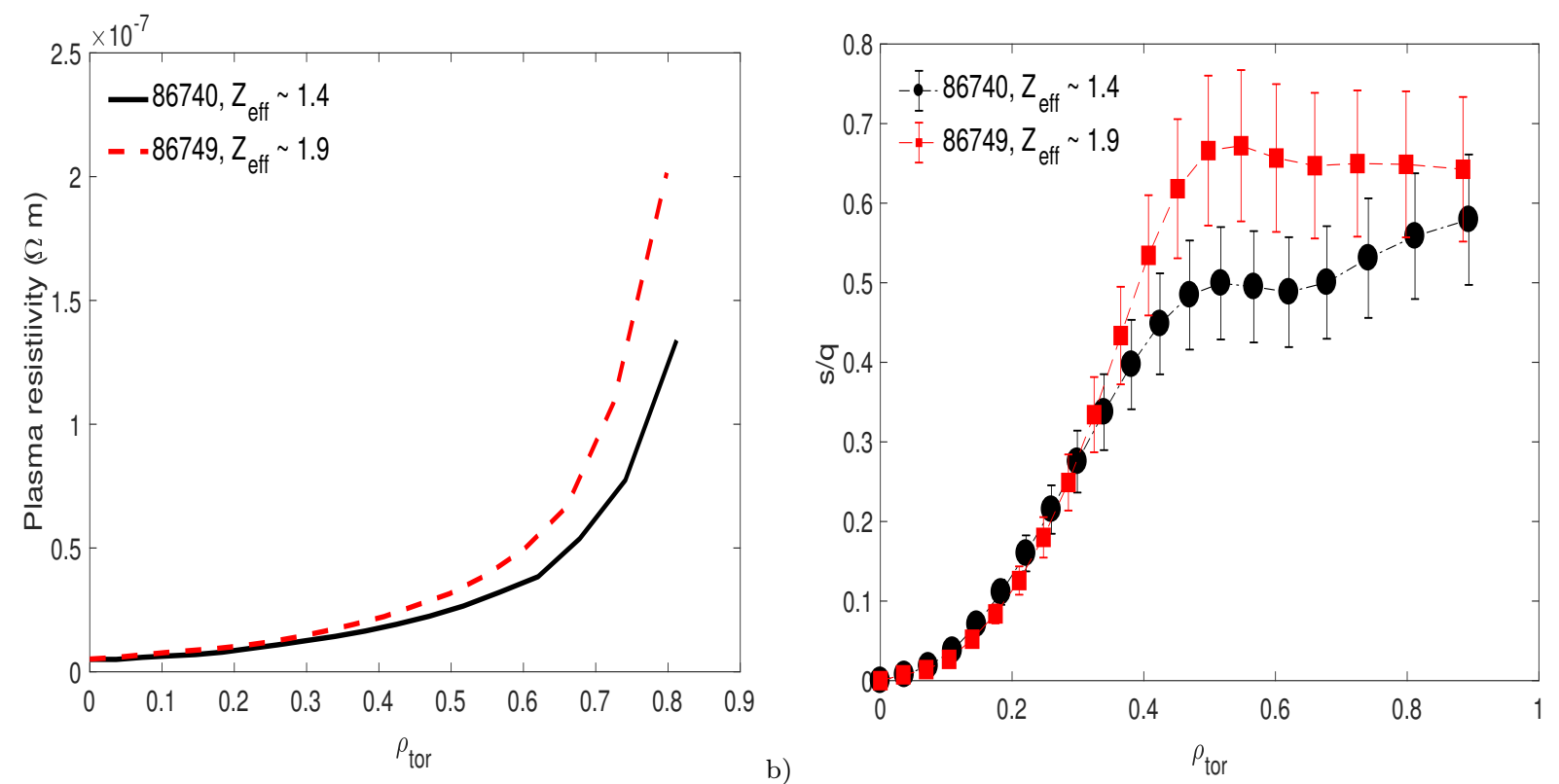

Figure 7: Radial profiles of the plasma resistivity (a) and $s / q$ (b) of the JET discharges $n .86740$ at $t=10 \mathrm{~s}$ (black circles) and n. 86749 at $t=9 \mathrm{~s}$ (red squares). Discharge $n .86740$ had $Z_{\text {eff }} \approx 1.4$, while discharge $n .86749$ had $Z_{\text {eff }} \approx 1.9$. 

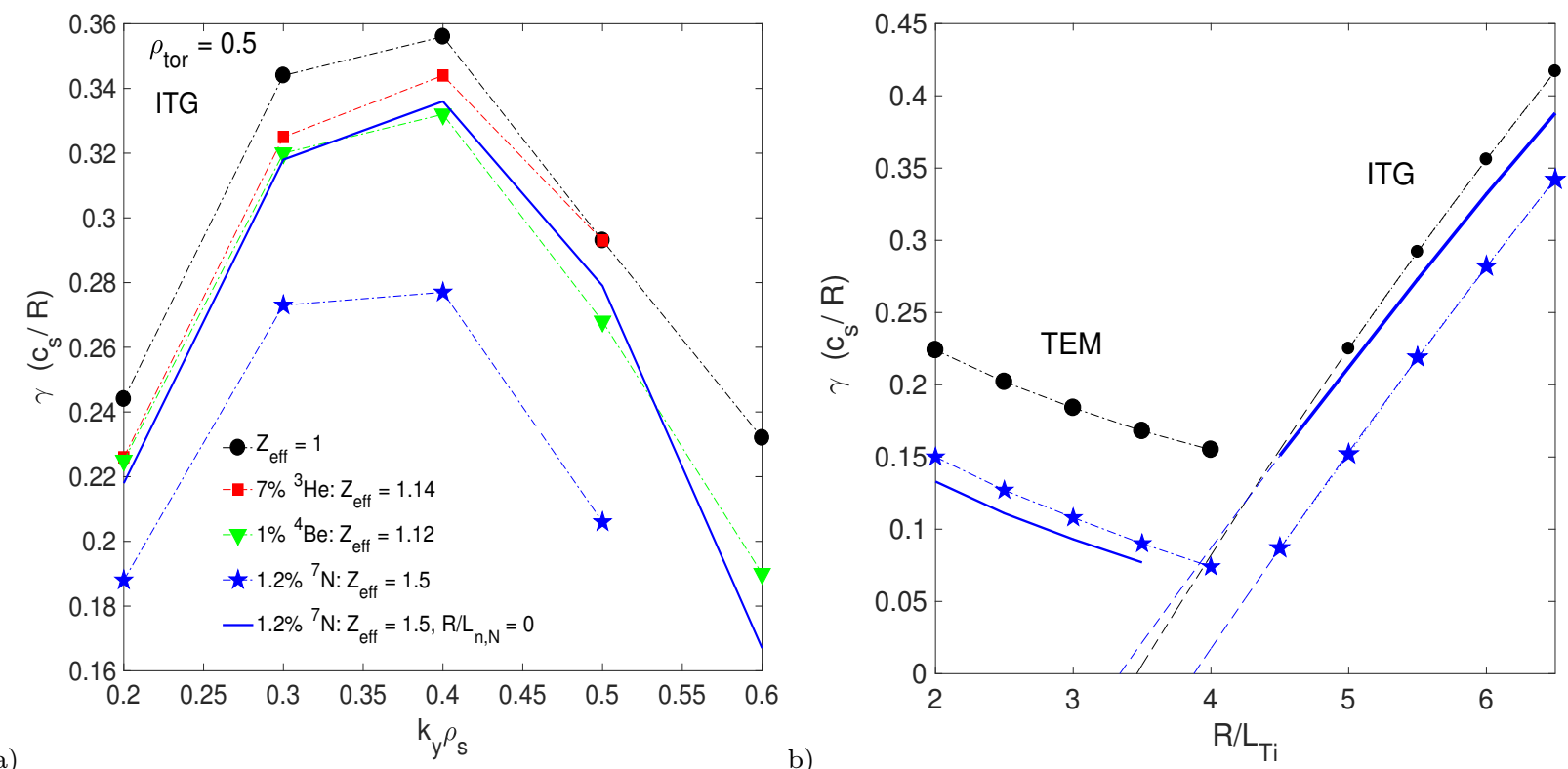

Figure 8: Normalized linear growth rate $\gamma$ of the main micro-instability from linear gyro-kinetic simulations at $\rho_{\text {tor }}=0.5$. a) $\gamma$ as a function of $k_{y} \rho_{s}$ : effects on the linear growth rate of ITG of $N,{ }^{3} \mathrm{He}$ and Be at experimental level using $R / L_{n, Z}=2.7$. The blue continuos line indicates the case with $N, R / L_{n Z}=0$. b) $\gamma$ as a function of $R / L_{T i}\left(k_{y} \rho_{s}=0.33\right)$ : effects on the linear growth rate of $N$ using $R / L_{n, Z}=2.7$ (blue pentagrams) and $R / L_{n Z}=0$ (continuos blue line). 

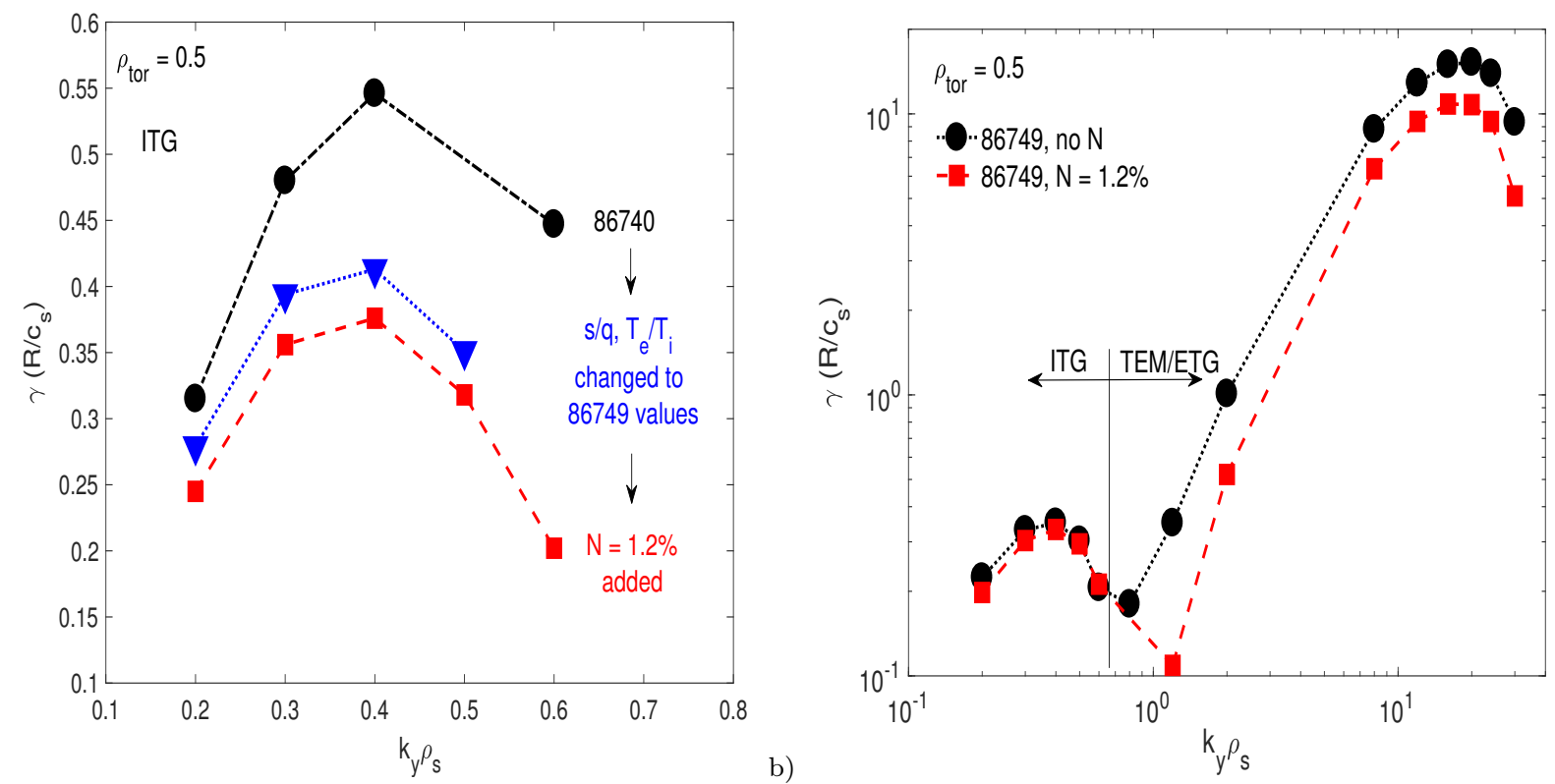

a)

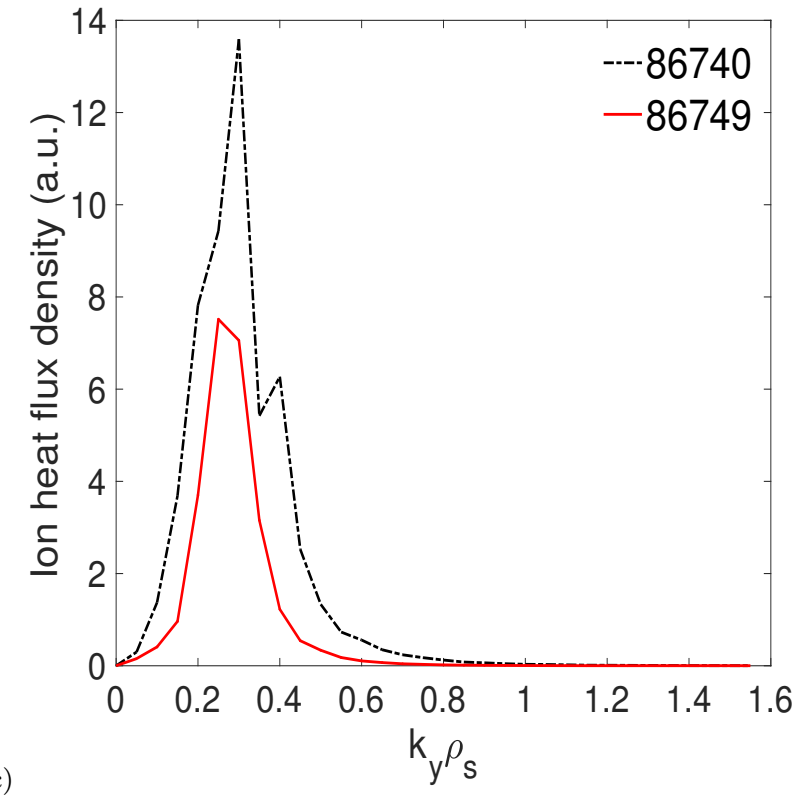

Figure 9: Normalized linear growth rate $\gamma$ of the main micro-instability as a function of $k_{y} \rho_{s}$ from linear gyro-kinetic simulations at $\rho_{\text {tor }}=0.5$. These results have been obtained using input parameters from shot $n$. 86740 (black circles), changing the values of $T_{e} / T_{i}$ and of $s / q$ to the ones of discharge $n .86749$ (blue triangles) and then adding $1.2 \%$ of nitrogen (red squares). the results covering both ion and electron range of $k_{y}$ are shown in figure (b). c) Ion heat flux density as a function of $k_{y} \rho_{s}$ for the case $R / L_{T i} \approx 5$ at $\rho_{\text {tor }}=0.5$. 

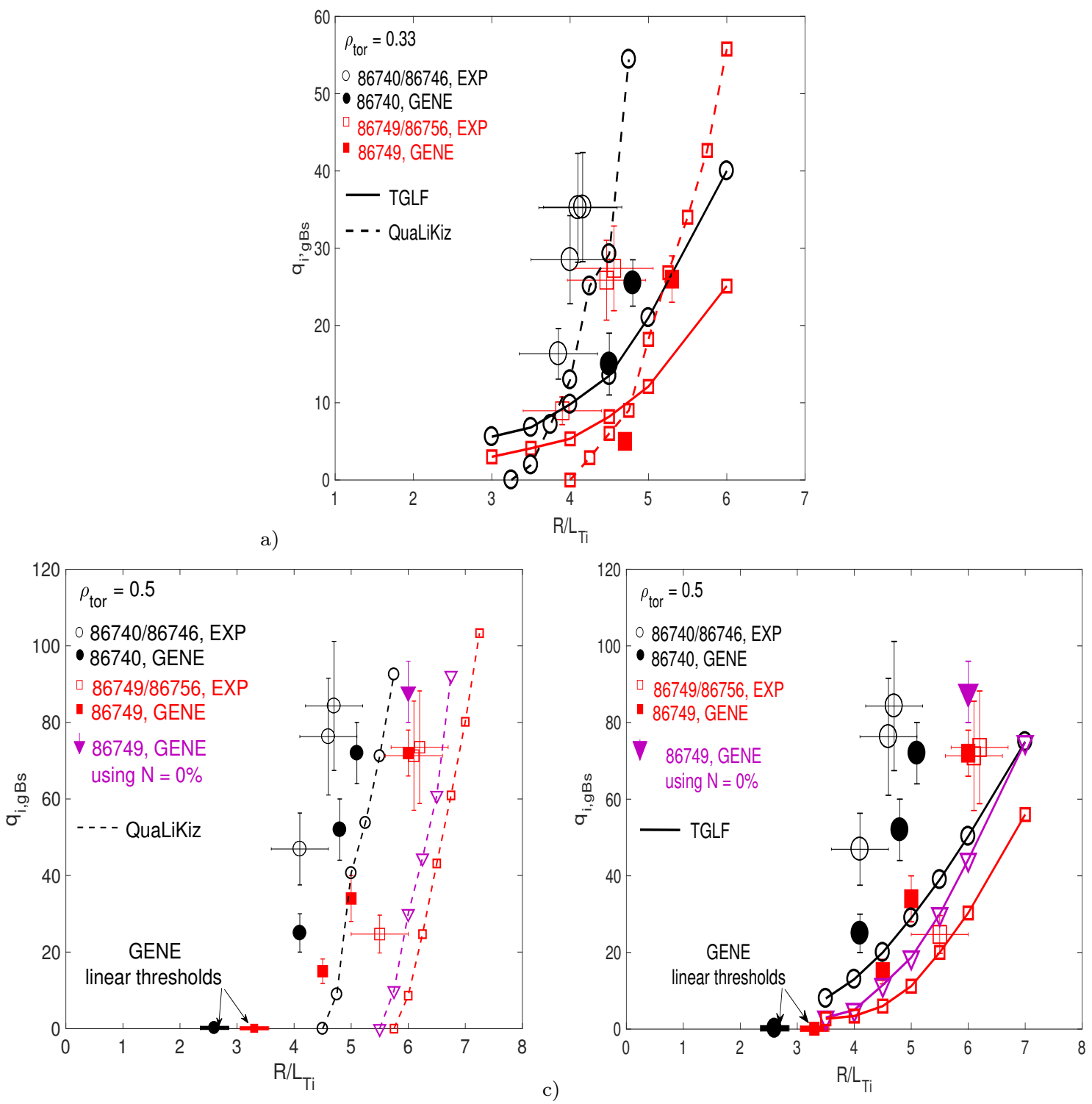

Figure 10: $q_{i, g B s}\left(R / L_{T i}\right)$ at $\rho_{t o r}=0.33$ (a) and $\rho_{t o r}=0.5(b, c)$. The black circles indicate the results for discharge n. $86740(N=0 \%)$ while the red squares indicate the results for discharge $n$. $86749(N=1.2 \%)$. The empty symbols represent the experimental results, the full symbols represent the nonlinear gyro-kinetic simulations, the dotted lines represent the QuaLiKiz simulations and the continuous lines represent the TGLF simulations. In figure (b) and (c) the purple triangles represent the simulations of discharge n. 86749 but using $N=0 \%$. 

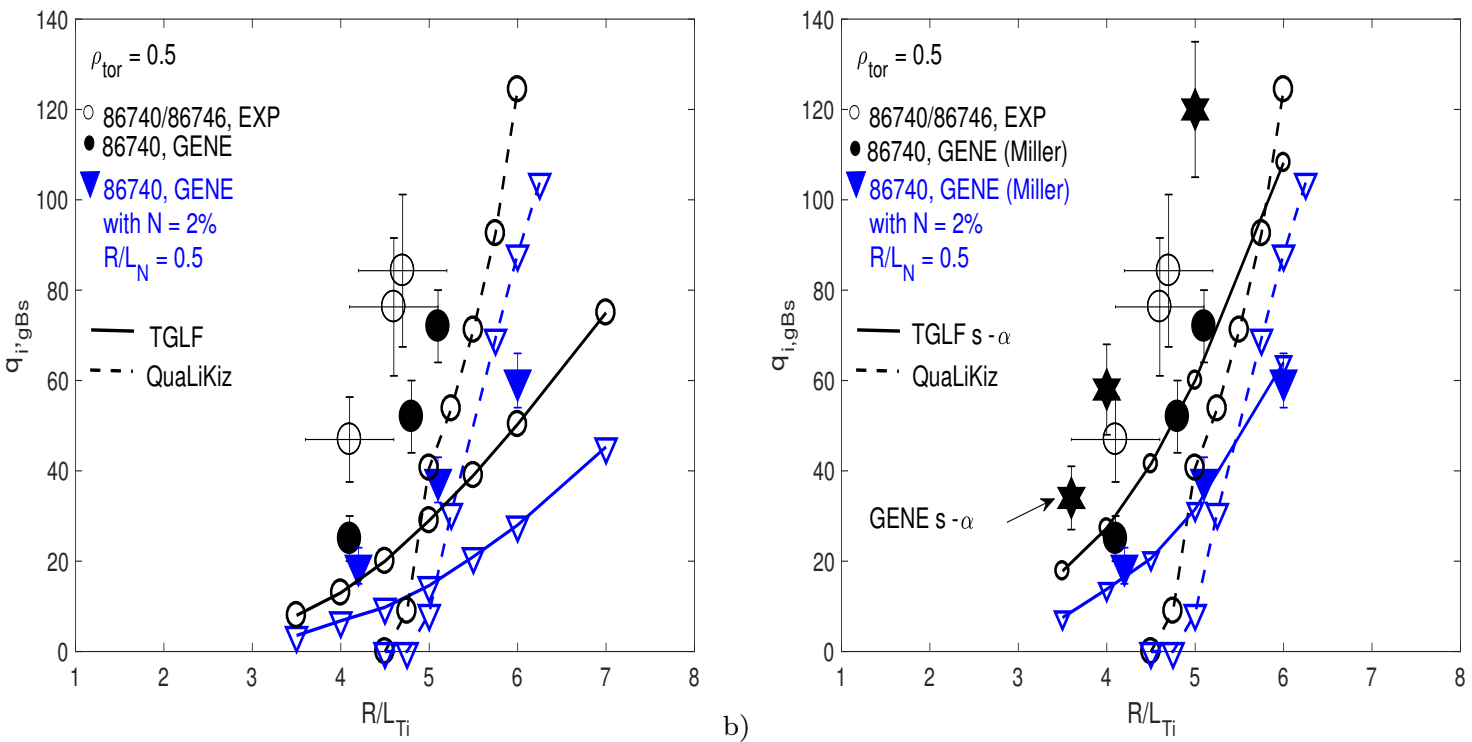

Figure 11: $q_{i, g B s}\left(R / L_{T i}\right)$ at $\rho_{\text {tor }}=0.5$. The black circles indicate the results for discharge n. $86740(N=0 \%)$. The empty symbols represent the experimental results, the full symbols represent the nonlinear gyro-kinetic simulations, the dotted lines represent the QuaLiKiz simulations and the continuous lines represent the TGLF simulations. The blue triangles represent the simulations of discharge $n .86740$ but using $N=2 \%, R / L_{N}=0.5$. In figure (b) the black hexagons represent GENE simulations for discharge n. 86740 but using $s-\alpha$ geometry instead of Miller geometry. 